\title{
Panchromatic Satellite Image Classification for Flood Hazard Assessment
}

\author{
Ahmed Shaker ${ }^{*}$, Wai Yeung Yan ${ }^{1}$, Nagwa El-Ashmawy ${ }^{1,2}$ \\ ${ }^{1}$ Department of Civil Engineering, Ryerson University, Toronto, Ontario, Canada \\ ${ }^{2}$ Survey Research Institute, National Water Research Center, Cairo, Egypt \\ *ahmed.shaker@ryerson.ca
}

\begin{abstract}
The study aims to investigate the use of panchromatic (PAN) satellite image data for flood hazard assessment with an aid of various digital image processing techniques. Two SPOT PAN satellite images covering part of the Nile River in Egypt were used to delineate the flood extent during the years 1997 and 1998 (before and after a high flood). Three classification techniques, including the contextual classifier, maximum likelihood classifier and minimum distance classifier, were applied to the following: 1) the original PAN image data, 2) the original PAN image data and grey-level co-occurrence matrix texture created from the PAN data, and 3) the enhanced PAN image data using an edgesharpening filter. The classification results were assessed with reference to the results derived from manual digitization and random checkpoints. Generally, the results showed improvement of the calculation of flood area when an edge-sharpening filter was used. In addition, the maximum likelihood classifier yielded the best classification accuracy (up to $97 \%$ ) compared to the other two classifiers. The research demonstrates the benefits of using PAN satellite imagery as a potential data source for flood hazard assessment.
\end{abstract}

Keywords: Panchromatic imagery, flood hazard assessment, texture analysis, image classification.

\section{Introduction}

Commercial remote sensing satellite sensors such as GeoEye, IKONOS, QuickBird and Worldview acquire panchromatic (PAN) and multispectral (MS) satellite images simultaneously. Recently, the rapid advancement in satellite sensor development enhances the capability in image acquisition with improved spatial, spectral and temporal resolutions [1]. This can be noticed in the radiometric resolution of the recently launched satellite sensor, for instance Worldview-2, where four more spectral bands, including coastal $(400-450 \mathrm{~nm})$, yellow $(585-625 \mathrm{~nm})$, red-edge $(705-745 \mathrm{~nm})$ and infrared red (IR), can be found in addition to the existing red, green, blue and near IR bands. The spatial resolution of Worldview-2 reaches $0.46 \mathrm{~m}$ and 1.84 $\mathrm{m}$ (at nadir) in PAN mode and MS mode, respectively [2]. By measuring the electromagnetic radiation recorded from the Earth surface across visible to infrared spectrum, MS imagery is particularly useful for scientific modeling in physical, biological, and social science studies. Examples are not limited to monitoring land use/land cover changes [3], natural hazards assessment [4], surface moisture monitoring [5], vegetation mapping [6], and ecological studies [7].
Unlike MS imagery, applications of PAN imagery mainly rely on its high spatial resolution for topographic information extraction. Various applications of PAN imagery can be found in the studies of panchromatic and multi-spectral image fusion [8][9], topographic mapping and digital elevation modeling [10], feature detection and extraction [11][12][13], and stereo data extraction [14]. Since the recorded spectral reflectance of PAN imagery is the total electromagnetic radiation recorded across the visible to part of the near-IR spectral range (commonly between 400 to $900 \mathrm{~nm}$ ), therefore, the reflectance values recorded by a sensor of specific land cover feature are mixed up. In addition, between-class spectral confusion and within-class spectral variation pose considerable challenges in land cover classification and object recognition, particularly in high resolution satellite imagery [15]. Therefore, the use of radiometric (spectral) information of PAN imagery is limited. To address this issue, ongoing researches were conducted to incorporate the spatial coherence in either the feature spaces or the classification algorithms to 
improve the performance of feature extraction and image classification of PAN imagery [11][15][16].

Since water bodies are found to have low spectral reflectance in the PAN spectral range compared to other ground features (see Figure 1), preliminary investigations using SPOT PAN satellite images were conducted to delineate flood area along the Nile River using pixel-based image classification and texture analysis [17]. Although the overall accuracy of classification results was high ( 90\%), the delineated flood area from different classification techniques had large variations compared to the reference data [17]. This phenomenon can be explained by the use of too many textures which dominate the feature spaces in the classification problem. Such strategy is only recommended for solving heterogeneous classification tasks [13][18].

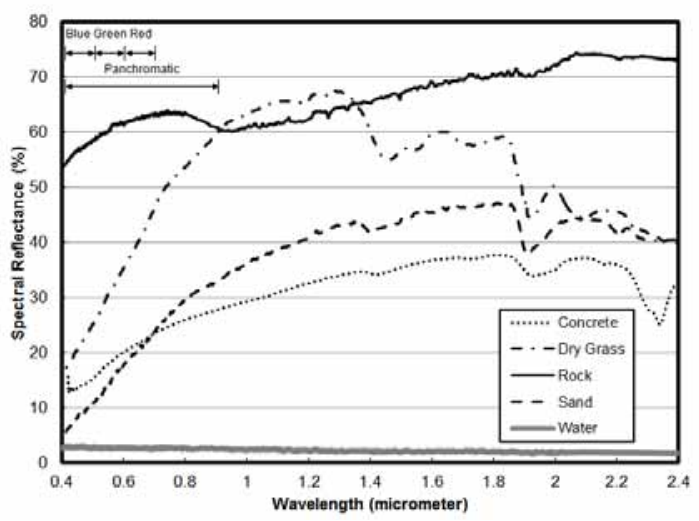

Figure 1. Spectral reflectance of different materials extracted from the NASA ASTER spectral library [19]; water bodies always have lower spectral reflectance than the other ground materials/features.

In this study, similar experiments were performed by enlarging the study area along the Nile River in order to solidify the results and the conclusions of PAN image classification as presented in [17]. The edge-sharpening (ES) filter was applied to enhance the PAN images before conducting classification. The purpose of applying the ES filter is to enhance the contrast of PAN images and highlight the edges of the water bodies from the surrounding features. Since the performance of the canny edge detector, k-nearest neighbor classifier and artificial neural network classifier were ordinary in the previous study [17], these techniques were not incorporated in this study. Instead, the contextual classifier, minimum distance classifier, and maximum likelihood classifier were utilized to classify the original PAN image, the enhanced PAN image with the ES filter, and the PAN image with grey level co-occurrence matrix (GLCM) texture. A comparison between the results derived from manual digitization and image classification techniques was conducted to evaluate the accuracy of the delineated flood area. Finally, accuracy assessment was carried out on the classification results with reference to a number of random checkpoints.

\section{Study area and data}

Nile River, regarded as the longest river in the world, faced flood problem every year. The river passes through ten African countries which are affected by the river flood during the rainy season. Egypt used to face the Nile River floods peacefully after building the Aswan High Dam in the South of Egypt. Yet, for the safety of the Aswan High Dam, releasing a large discharge of water was one of the few scenarios that have been used to deal with consecutive years of high floods. Another scenario was flushing exceeding water into depressions in the Western Desert (Toshka Depression). However, releasing a large amount of water from the Aswan High Dam to the Nile River affects some of the Nile islands and inundates some of the Nile River banks.

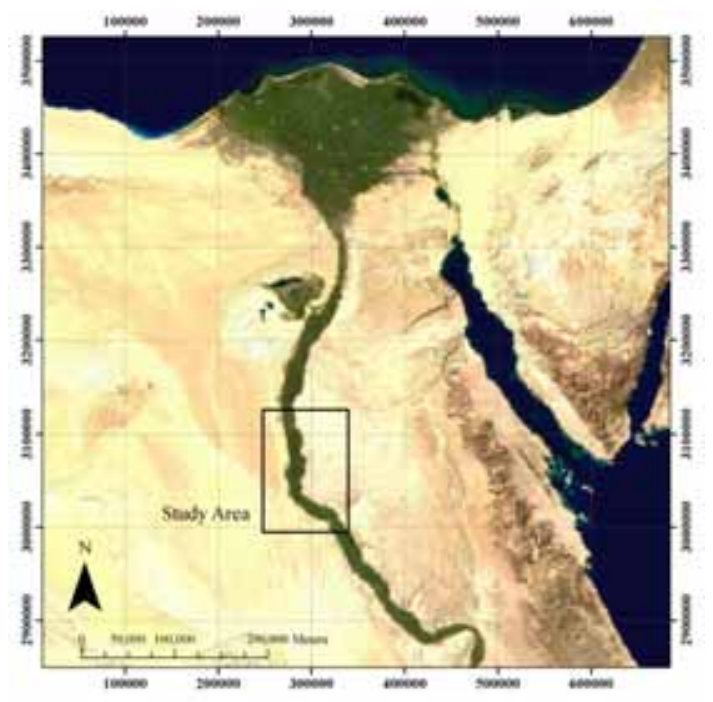

Figure 2. Study area along the Nile River. 
This study investigates the potential use of PAN satellite imagery for flood hazard assessment. The study area is located along a central part of the Nile River in Egypt (at $29^{\circ} 16$ 'N, 31 $17^{\circ}$ ' E). Figure 2 shows the location of the study area. Two cloud-free SPOT PAN satellite images covering the study area in the years 1997 and 1998 were acquired before and after the $1997 / 1998$ high flood. The spatial, spectral and radiometric resolutions of the two images are 10 $\mathrm{m}, 0.51-0.73 \mu \mathrm{m}$ and 8 bit, respectively.

\section{Methodology}

\subsection{Overall workflow}

Figure 3 shows the experimental workflow of the study. Two SPOT PAN satellite images acquired before and after the flood were used. Due to the lack of ground control points, image-to-image registration was first conducted in order to align both images in the same image coordinate system.

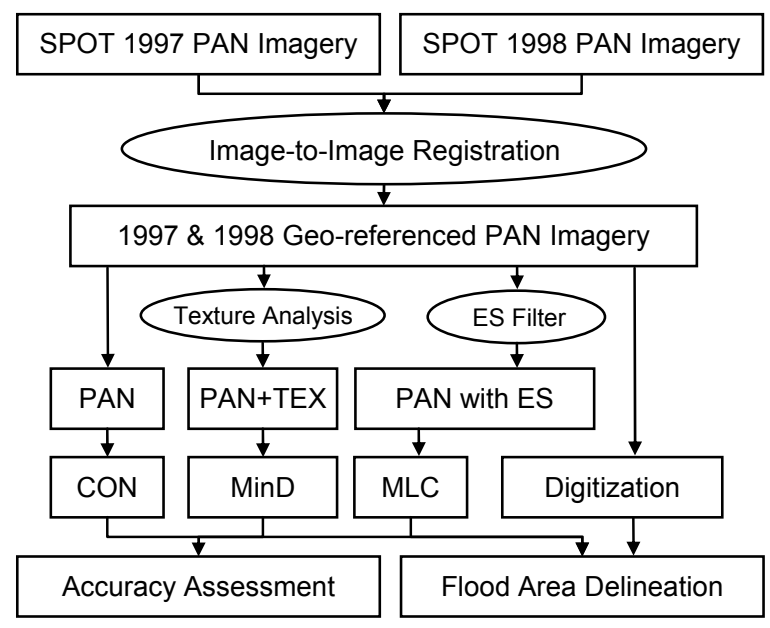

Figure 3. Experimental workflow.

The registered PAN images were used to go through two processes: a) texture analysis, and b) enhancement with ES filter. Three datasets (original PAN images, PAN image with TEX, and PAN images applied with ES filter) were classified using three different image classification techniques. Finally, accuracy assessment was carried out on each of the classification results with an aid of random checkpoints, and the flood area delineated from the image classification techniques were compared to the flood area derived from manual digitization. Steps of the workflow are described in the following sections.

\subsection{Image-to-image registration}

Due to the lack of ground control points and digital elevation model, orthorectification cannot be achieved on the two PAN images. Therefore, image-to-image registration was conducted so that an identical image coordinate system could be established for the two SPOT images. The geometric distortion of the two images can be ignored because of the minimum changes in the image viewing angle and elevation differences along the Nile River. By digitizing 40 control points, the year 1998 PAN image was registered to the year 1997 PAN image based on a second-order polynomial model. The error of image-to-image registration was 1.26 pixels in $X$ direction and 0.33 pixel in $Y$ direction, and the overall root-meansquared error was 1.33 pixels. Figure 4 shows the SPOT PAN image acquired in the years 1997 (before flood) and 1998 (after flood) after the image-to-image registration process.

\subsection{Texture analysis}

Texture analysis is one of the proven methods to improve the land cover classification accuracy [21]. Texture analysis takes into consideration of the distribution and variation of neighborhood pixel data. Hence, the spatial properties of land cover classes can be incorporated as one of the classification criterion. Comprehensive literature review of texture analysis for PAN satellite images can be found in [15][18][22]. Texture analysis can be categorized into structural level textures and statistical level textures. This study focuses on the latter approach since it is more suitable for classification of scenes covering natural features [18]. In the statistical approach, the stochastic properties of the spatial distribution of grey level $(\mathrm{GL})$ in the image are characterized. Amongst all popular algorithms, grey level co-occurrence matrix (GLCM) is a widely adopted method [23]. The first order and second order texture measures of GLCM consist of standard deviation (SD), range, minimum, maximum and mean. The second order of texture measures includes angular second moment, contrast, correlation, dissimilarity, entropy, information measures of correlation, inverse difference moment and sum of squares variance. 

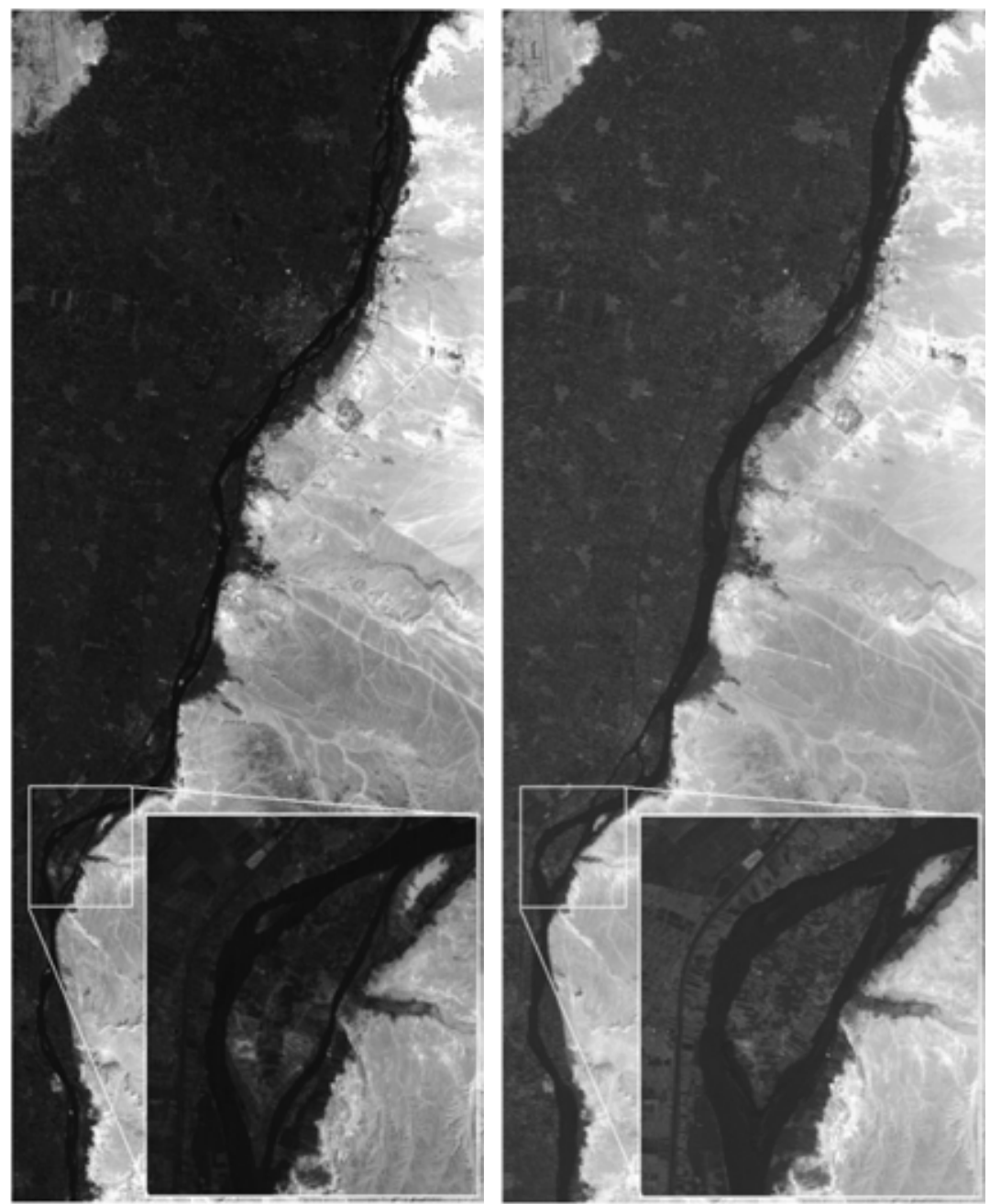

Figure 4. Panchromatic SPOT images acquired in the years 1997 (left) and 1998 (right).

Although the majority of these textures could be found in commercial remote-sensing software packages, the use of GLCM textures still require the user's decision on a number of parameters including spatial resolution, spectral band, quantization level of the image, size of the moving windows, and inter-pixel distance and angle during the co-occurrence computation [21].

By visually comparing both SPOT PAN images, water bodies, desert areas and other lands were distinguishable; however, farmlands and water bodies were found to be mixed up with similar pixel values. Based on our previous study [17], it was shown that incorporation of too many texture measures dominated the feature spaces in the classification problem leading to inconsistent results. Therefore, in this study, only the GLCM mean texture was incorporated in this study since it was proven to be the most useful texture [15]. The mean texture can be computed from the cooccurrence matrix of the image by using the following Equation (1).

$$
\text { Mean }=\sum_{x=0}^{N-1} \sum_{y=0}^{N-1} x P_{x, y}
$$

where $P$ is the normalized symmetric GLCM of dimension $G \times G, G$ is the number of grey levels and $P_{x, y}$ is the element of $P$ in the $x^{\text {th }}$ row and the $\mathrm{y}^{\text {th }}$ column. 
According to [21] and [24], the window size for texture analysis should be smaller than the smallest object to be mapped in the image. In this study, a $5 \times 5$ window size was selected which was able to capture the textural characteristics, especially those small isolated island along the Nile River. Regarding the orientation, we adopted the setting of $0^{\circ}, 45^{\circ}, 90^{\circ}$ and $135^{\circ}$ which should be able to cover all directions of Nile River and the islands in the PAN images. Finally, the inter-pixel distance was set to be 1 in this study since there was no significant difference between 1 and 8 in [25] and between 1 and 4 in the previous study [18]. Higher inter-pixel distance may even decrease the overall classification accuracy as revealed from these studies. Finally, the generated GLCM mean texture was exported as a 32-bit image band which was incorporated with the SPOT PAN image for classification.

\subsection{Edge-sharpening (ES) filter}

The ES filter was applied on the original PAN image in order to enhance the boundary of the water bodies from the coastal area. The principle of ES filter is to first subtract the original image from an average filtered image. The difference between these two images is then added back to the original image in order to enhance the high frequency features. Figure 5 shows the SPOT PAN image before and after applying the ES filter. The ES filter was applied to the two SPOT images (before and after the flood) and then pixel-based image classification was carried out. Polygon-based training sites were identified for three different land cover classes: agricultural land (class 1), desert area (class 2) and water bodies (class 3). Then, three supervised classification methodologies (CON, MinD, and MLC) were used to classify the three stated classes using the same training samples.

\subsection{Image classification techniques}

The study focuses on three different classification techniques which are the contextual classifier (CON), maximum likelihood classifier (MLC), and minimum distance classifier (MinD) since they are commonly built in existing image processing software. The three techniques were applied to the original PAN satellite image, the original PAN image and GLCM texture, and the enhanced PAN image using the ES filter. The MLC technique relies on the computation of the probability density function for the training statistics of each land cover class. The estimated probability function for class $w_{i}$ can be determined for $n$ dimensional space (or bands) using Equation 2 [20],

$$
p\left(X \mid w_{i}\right)=\frac{1}{(2 \pi)^{\frac{n}{2}}\left|V_{i}\right|^{\frac{1}{2}}} \exp \left[-\frac{1}{2}\left(X-M_{i}\right) V_{i}^{-1}\left(X-M_{i}\right)\right]
$$

where $n$ is the number of bands, $V_{i}$ is the covariance matrix for each class, $M_{i}$ is the mean vector of each class, $X$ refers to the pixel value of the image data for the $n$ bands. All these input data can be computed when the training sites are identified for each class.
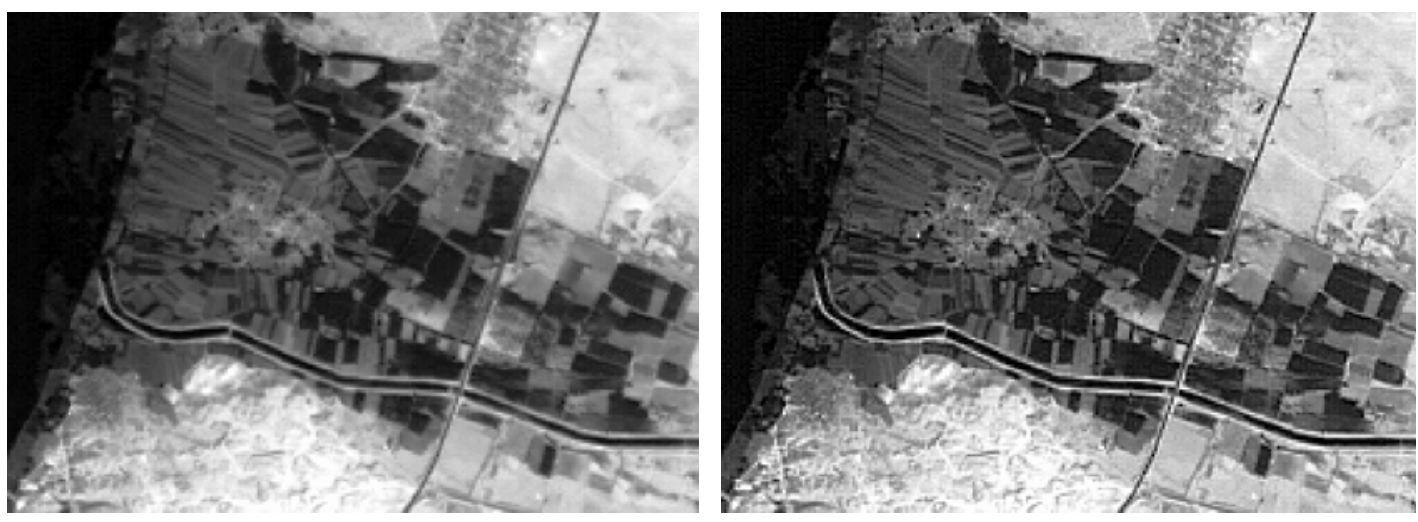

Figure 5. Original SPOT PAN Image (left) and SPOT PAN Image applied with ES Filter (right). 
After computing the probability function for all the classes, the maximum likelihood decision rule decides $X \in w_{i}$ if and only if

$$
p\left(X \mid w_{i}\right) \cdot p\left(w_{i}\right) \geq p\left(X \mid w_{j}\right) \cdot p\left(w_{j}\right)
$$

for all $i$ and $j$ out of $1,2, \ldots m$ possible classes. Therefore, pixel $X$ will be assigned the class $w_{i}$ with the largest probability density function.

The MinD classification technique does not consider the probability density function for each class. Instead, it computes the distance between pixel value $X$ of the image data and the mean value of class $w_{i}$ from the training sites using Equation 4 [20],

$$
D_{x, w_{i}}=\sqrt{\sum_{i=1}^{n}\left(X-\mu_{n, i}\right)^{2}}
$$

where $\mu_{n, i}$ is the mean value of the land cover class $w_{i}$ in the $n^{\text {th }}$ band, and the pixel value $X$ will be assigned to the class $w_{i}$ if and only if

$$
D_{x, w_{i}} \leq D_{x, w_{j}}
$$

for all $i$ and $j$ out of $1,2, \ldots m$ possible classes.

The CON classification technique performs a frequency-based classification on the PAN imagery with two major steps [26]. Since the PAN image is a single band grey-level image, reduction algorithm may not be necessary. Occurrence frequency $f$ is computed with respect to the number of times that a specific pixel $X$ appears in a defined window centered at $x, y$. The window is then looped for the entire image and a frequency band is generated. Additional statistical measures such as mean, SD, skewness, kurtosis, range, and entropy can be derived from the frequency band. Classification can then be conducted on the frequency band(s) as well as the PAN imagery using any parametric classifier such as MinD.

The results of the three approaches were presented and assessed with the aid of the results derived from manual digitization. For manual digitization, the two SPOT PAN images were imported into ESRI® ArcGIS ${ }^{\text {TM }}$ ArcMap for digitizing the boundary of the Nile River before and after the flood. Small islands appearing along the river were delineated; as a result, the output of the river would be in a form of polygon with holes. After digitization, the GIS polygon layer representing the digitized water zones of the Nile River of the year 1998 was clipped by the polygon layer representing the digitized water zones of the year 1997. The resulting GIS polygon layer thus represented the flood zones. The area of these zones was calculated as a reference to assess the results of image classification.

The classification accuracy was evaluated using 200 checkpoints randomly distributed in the study area. The overall accuracy and kappa statistics were computed. Finally, the flood zones from the classification results were calculated by subtracting the classification results of the year 1997 from the classification results of the year 1998. Three rounds of classification were carried out for the three datasets (original PAN images, enhanced PAN images using ES filter, original PAN images GLCM texture) to compare the accuracy of the flood area delineation using different classification techniques.

\section{Results and analysis}

Figures $6 a$ to $6 \mathrm{j}$ show the flood area extracted after applying various image classification techniques as follows: 1) Figures $6 \mathrm{a}, 6 \mathrm{~d}$ and $6 \mathrm{~g}$ show the results of the original PAN image classification using $\mathrm{CON}$, MinD and MLC, respectively; 2) Figures 6b, 6e and $6 \mathrm{~h}$ show the results of the enhanced PAN image classification using $\mathrm{CON}, \mathrm{MinD}$ and MLC, respectively; 3) Figures $6 \mathrm{c}, 6 \mathrm{f}$ and $6 \mathrm{i}$ show the image classification results of the original PAN images integrated with textures using CON, MinD and MLC, respectively. By visually comparing the results, most of the delineated flood area were close to the results derived from manual classification (Figure 6j), except the result of CON classifier on PAN + TEX images (Figure 6g) which demonstrated more flood area in the islands along the Nile River.

Table 1 shows the flood areas computed from the image classification techniques as explained previously. The flood areas calculated by manual digitization method was found to be $13.15 \mathrm{~km}^{2}$ and it was used as a reference to evaluate the accuracy 
of other classification techniques. The values between the brackets show the percentage difference between the results of manual digitization and the results from the corresponding image classification technique.

\begin{tabular}{|c|c|c|c|}
\hline & CON & MinD & MLC \\
\hline PAN & $\begin{array}{c}13.74 \\
(+4.5 \%)\end{array}$ & $\begin{array}{c}15.50 \\
(+17.9 \%)\end{array}$ & $\begin{array}{c}12.50 \\
(-4.9 \%)\end{array}$ \\
\hline PAN with ES & $\begin{array}{c}13.74 \\
(+4.5 \%)\end{array}$ & $\begin{array}{c}14.51 \\
(+10.4 \%)\end{array}$ & $\begin{array}{c}13.36 \\
(+1.7 \%)\end{array}$ \\
\hline PAN + TEX & $\begin{array}{c}20.39 \\
(+55.2 \%)\end{array}$ & $\begin{array}{c}15.56 \\
(+18.4 \%)\end{array}$ & $\begin{array}{c}12.55 \\
(-4.5 \%)\end{array}$ \\
\hline
\end{tabular}

Table 1. Flood area delineation from different image processing techniques (in $\mathrm{km}^{2}$ ).

It was found that the area derived from MLC yielded the best results when the classifier was applied to the enhanced PAN images with the ES filter (+1.7\% difference only). MLC delineated less flood area $(\sim-5 \%)$ in cases where original PAN image and PAN+TEX images were used. For the flood areas delineated by MinD, all the results were greater than the actual flood area calculated by manual digitization ranging from $10 \%$ to $20 \%$. However, the results of MinD classifier were improved by about $8 \%$ when the flood area was derived from the enhanced PAN images using the ES filter. This argument can also be observed when CON and MLC classifiers were applied to the PAN images enhanced by the ES filter.

When the CON classifier was applied to the original PAN images and enhanced PAN images, they produced slightly more flood area than the manual digitization approach by $4.5 \%$. Although the CON classifier was not as accurate as the MLC classifier in terms of overall accuracy (see Table 2), the results echoed our previous findings where the CON classifier worked well in delineating homogenous areas [17]. One should also note that there was a significant difference in the result of the CON classifier applied to PAN + TEX imagery ( $55.2 \%)$. This finding also aligned to our previous findings in [17] which can be explained by the similar rationale of the contextual classifier and the texture analysis. Both techniques require the computation of spatial features from the neighborhood pixels and this leads to a duplication of spatial coherence in the classification problem.

The overall accuracy obtained from the classification with inclusion of the original PAN image enhanced the PAN image with the ES filter, and both PAN + TEX images are presented in Table 2. The overall accuracy of the MLC was found always superior to the other classification results with accuracy ranging from $88 \%$ to $97 \%$ on both 1997 and 1998 dataset. The second accurate results were derived from the MinD with overall accuracy varying $83 \%$ to $87 \%$. However, CON did not perform well in terms of the classification accuracy. The CON classifier produced low classification accuracy $(\sim 50 \%)$ in most of the results, except the results of the year 1997 with PAN+TEX images and the PAN image enhanced with the ES filter (up to 90\%). Although the flood area derived from PAN image classification by the CON was close to the results of manual digitization, the overall accuracy was low because the agricultural lands (located at the west side of the Nile River) were misclassified into water bodies. Therefore, one can conclude that the CON classifier is suitable for homogeneous feature extraction which may not be very efficient in general for land cover map production. In terms of the dataset, classification of PAN + TEX images achieved slightly higher accuracy than the classification of the original PAN image and the classification of the enhanced PAN image with the ES filter, except the year 1997 image classified by the CON classifier. From the results obtained in MLC, it is proved that classification of the PAN+TEX image is useful for land cover mapping.

\begin{tabular}{|c|c|c|c|}
\hline \multicolumn{4}{|c|}{ SPOT 1997 } \\
\hline & CON & MinD & MLC \\
\hline PAN & $41.5 \%$ & $85.5 \%$ & $91.0 \%$ \\
\hline PAN with ES & $97.0 \%$ & $83.0 \%$ & $88.0 \%$ \\
\hline PAN + TEX & $92.5 \%$ & $88.0 \%$ & $97 \%$ \\
\hline \multicolumn{4}{|c|}{ SPOT 1998 } \\
\hline & CON & MinD & MLC \\
\hline PAN & $57.0 \%$ & $87.0 \%$ & $95.0 \%$ \\
\hline PAN with ES & $57.0 \%$ & $85.0 \%$ & $92.0 \%$ \\
\hline PAN + TEX & $57.5 \%$ & $87.0 \%$ & $96.5 \%$ \\
\hline
\end{tabular}

Table 2. Overall accuracy of image classification. 


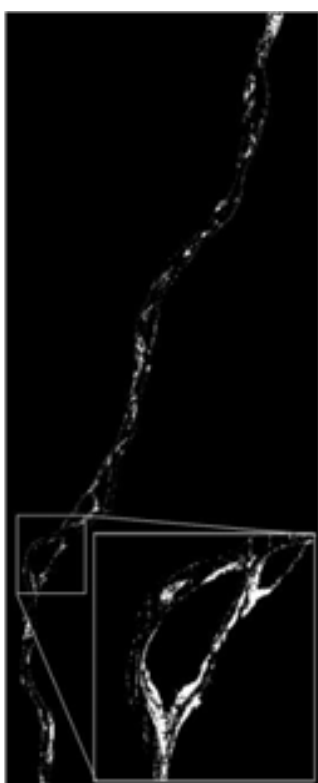

(a)

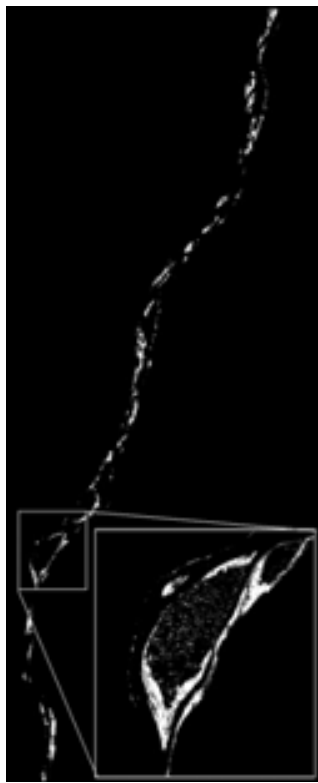

(f)

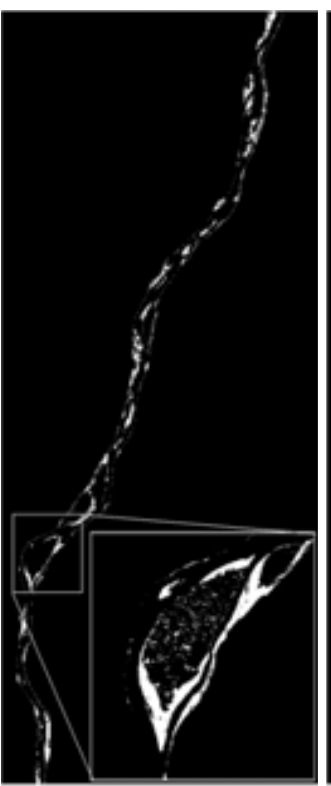

(b)

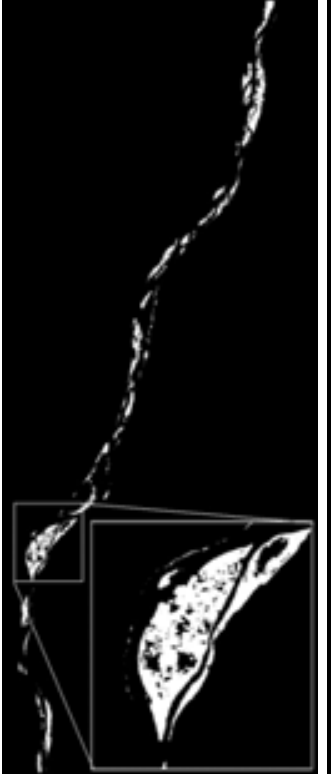

(g)

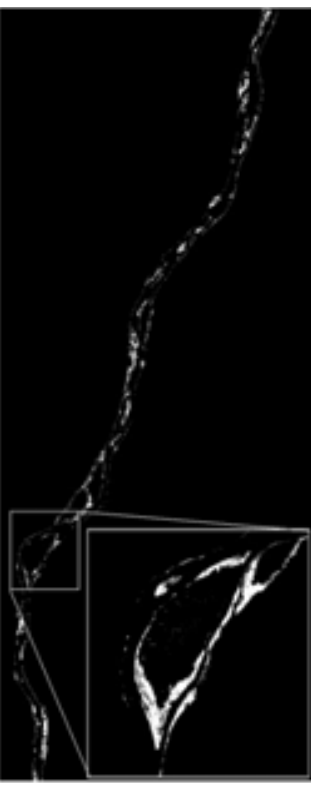

(c)

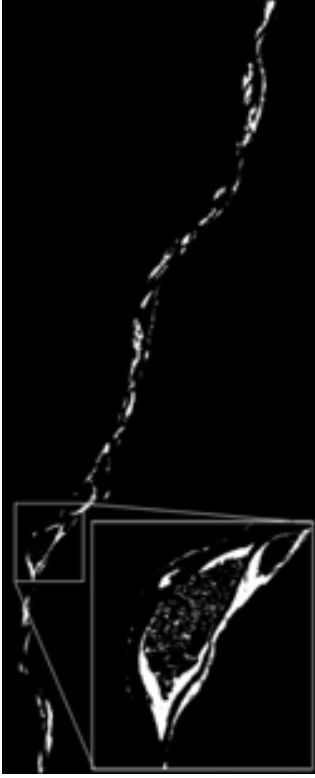

(h)

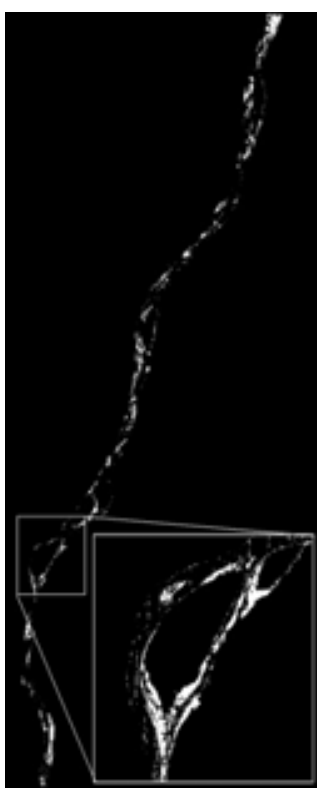

(d)

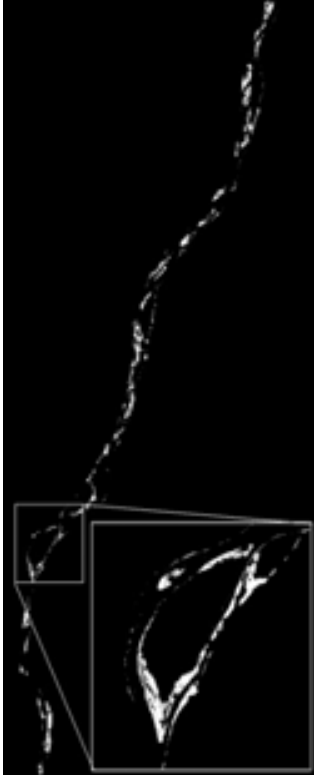

(i)

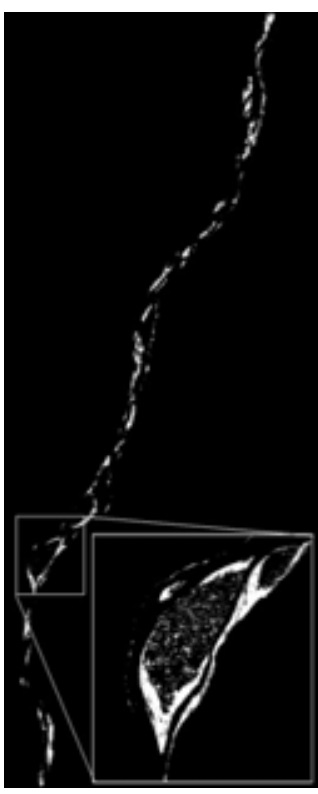

(e)

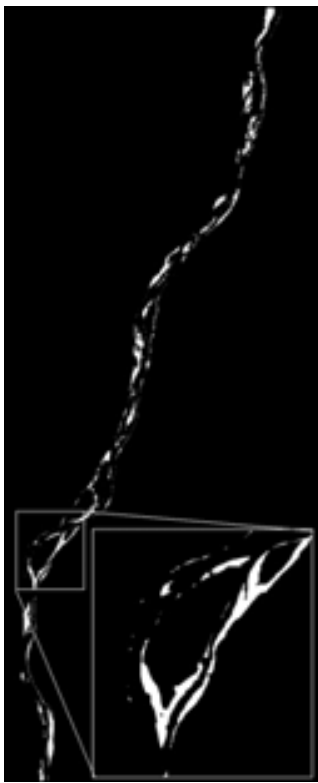

(j)

Figure 6. Flood area extracted from the PAN image using (a) CON, (b) MinD and (c) MLC; PAN image with ES filter using (d) CON, (e) MinD, (f) MLC; PAN+TEX images using (g) CON, (h) MinD, (i) MLC; and (j) manual digitization. 


\section{Conclusions}

This study demonstrates the potential use of PAN satellite imagery for flood hazard assessment with the aid of various image processing techniques. Two SPOT PAN satellite images, covering part of the Nile River in Egypt were acquired for the year 1997 (before flood) and the year 1998 (after flood) for experimental testing. Three image classification techniques were used: the contextual classifier, maximum likelihood classifier and minimum distance classifier. These classifiers were applied to three different forms of the data: original PAN satellite image, original PAN image and GLCM texture, and enhanced PAN image using the edgesharpening filter. The classification results were assessed with reference to the results derived from manual digitization and random checkpoints.

The results showed that MLC always produced the best results with the highest overall accuracy of $88 \%$ to $97 \%$. The flood area delineated by MLC was close to the results of manual digitization $( \pm 5 \%)$. It was also found that applying the edgesharpening filter on the PAN imagery leaded to an accuracy improvement of flood area delineation regardless of the classification techniques used. Generally, texture analysis can be incorporated for PAN image classification to compensate the lack of spectral information. However, too many textures may degrade the accuracy of image classification in homogeneous areas. The results also confirmed our previous findings [17] that a contextual classifier would be suitable for extracting homogenous features (i.e., water bodies) with shape edges. Since PAN satellite imagery has higher spatial resolution but lower acquisition cost than MS satellite imagery, PAN imagery can be used as a potential source of data for flood hazard assessment.

\section{Acknowledgements}

The findings presented in this paper are an extension to the results published in the International Conference and Exhibition MELAHA 2010 and the ISPRS 2008 Congress. The research work is supported by the Discovery Grant from the Natural Sciences and Engineering Research Council of Canada (NSERC).

\section{References}

[1] Benediktsson, J.A., Chanussot, J. \& Moon, W.M., Very High-Resolution Remote Sensing: Challenges and Opportunities. Proceedings of the IEEE, Vol. 100, No. 6, June, 2012, pp. 1907-1910.

[2] DigitalGlobe, Wordview-2 Satellite [online]. WWF http://www.digitalglobe.com WorldView-2, Accessed on November 2011.

[3] Rogan J. \& Chen D.M., Remote Sensing Technology for Mapping and Monitoring Land-cover and Land-use Change, Progress in Planning, Vol. 61, No. 4, May, 2004, pp. 301-325.

[4] Joyce K.E., Belliss S.E., Samsonov S.V., McNeill S.J. \& Glassey P.J., A Review of the Status of Satellite Remote Sensing and Image Processing Techniques for Mapping Natural Hazards and Disasters, Progress in Physical Geography, Vol. 33, No. 2, April, 2009, pp. 183-207.

[5] Wang L. \& Qu J.J., Satellite Remote Sensing Applications for Surface Soil Moisture Monitoring: A Review, Frontiers Earth Science in China, Vol. 3, No. 2, June, 2009, pp. 237-247.

[6] Xie Y., Sha Z. \& Yu M., Remote Sensing Imagery in Vegetation Mapping: A Review, Journal of Planet Ecology, Vol. 1, No. 1, March, 2008, pp. 9-23.

[7] Sacristán-Romero F., Technological Support for Ecology. Journal of Applied Research and Technology, Vol. 5, No. 3, December, 2007, pp. 160-169.

[8] Simone G., Farina A., Morabito F.C., Serpico S.B. \& Bruzzone L., Image Fusion Techniques for Remote Sensing Applications, Information Fusion, Vol. 3, No. 1, March, 2002, pp. 3-15.

[9] Zhang Y., Understanding Image Fusion, Photogrammetric Engineering \& Remote Sensing, Vol. 70, No. 6, June, 2004, pp. 657-661.

[10] Shaker A., Shi W. Z. \& Emam, H., The Use of Empirical Methods in Topographic Map Production of IRS-1D Images, The Annual Conference of American Society of Photogrammetry and Remote Sensing, Anchorage, Alaska, USA, May 5-9, 2003.

[11] Segl L. \& Kaufmann H., Detection of Small Objects from High Resolution Panchromatic Satellite Imagery based on Supervised Image Segmentation, IEEE Transactions on Geoscience and Remote Sensing, Vol. 39, No. 9, September, 2001, pp. 2080-2083. 
[12] Luo J., Ming D., Liu W., Shen Z., Wang M. \& Sheng H., Extraction of Bridges over Water from IKONOS Panchromatic Data, International Journal of Remote Sensing, Vol. 28, No. 16, August, 2007, pp. 3633-3648.

[13] Corbane C., Marre F. \& Petit M., Using SPOT-5 HRG Data in Panchromatic Mode for Operational Detection of Small Ships in Tropical Area, Sensors, Vol. 8, No. 5, May 2008, pp. 2959-2973.

[14] Shaker A., Yan W.Y. \& Easa S.M., Using Stereo Satellite Imagery for Topographic and Transportation Applications: An Accuracy Assessment, GIScience and Remote Sensing, Vol. 47, No. 3, September, 2010, pp. 321-337.

[15] Zhang Q., Wang J., Gong P. \& Shi P., Study of Urban Spatial Patterns from SPOT Panchromatic Imagery Using Textural Analysis, International Journal of Remote Sensing, Vol. 24, No. 21, November, 2003, pp. 4137-4160.

[16] Tso B. \& Mather, P.M., Classification Methods for Remotely Sensed Data, Second Edition, CRC Press, 2009, pp. 376.

[17] Shaker A., Yan W.Y., Wong M. S., El-Ashmawy N. \& Haddad B. I., Flood Hazard Assessment using Panchromatic Satellite Imagery. The XXI Congress of the International Society for Photogrammetry and Remote Sensing, 2008, pp. 881-886, Beijing, China, July 3-11, 2008.

[18] Shaban M.A., \& Dikshit O., Improvement of Classification in Urban Areas by the use of Textural Features the Case Study of Lucknow City, Uttar Pradesh. International Journal of Remote Sensing, Vol. 22, No. 4, 2001, pp. 565-593.

[19] Baldridge A.M., Hook S.J., Grove C.I. \& Rivera G., The ASTER Spectral Library Version 2.0, Remote Sensing of Environment, Vol. 113, No. 4, April, 2009, pp. 711-715.

[20] Jensen, J., Introductory Digital Image Processing, 3/e, Prentice Hall, Upper Saddle River, New Jersey, ISBN 0-13-1453361-0: 526p, 2005.

[21] Marceau D.J., Howarth P.J., Dubois J-M. M. \& Gratton D.J., Evaluation of the Grey-Level CoOccurrence Matrix Method for Land Cover Classification using SPOT Imagery, IEEE Transactions Geoscience and Remote Sensing, Vol. 28, No. 4, July, 1990, pp. 513-519.
[22] Narasimha Rao P.V., Sesha Sai M.V.R., Sreenivas K., Krishna Rao M.V., Rao B.R.M., Dwivedi R.S. \& Venkataratnam L., Textural Analysis of IRS-1D Panchromatic Data for Land Cover Classification, International Journal of Remote Sensing, Vol. 23, No. 17, 2002, pp. 3327-3345.

[23] Haralick R. M., Dinstein I. \& Shanmugam K., Texture Features for Image Classification, IEEE Transactions on Systems, Man, and Cybernetics, Vol. SMC-3, No. 6, November, 1973, pp. 610-621.

[24] Baraldi A. \& Parmiggiani F., An Investigation of the Textural Characteristics Associated with Gray Level Cooccurrence Matrix Statistical Parameters, IEEE Transactions on Geoscience and Remote Sensing, Vol. 33, No. 2, March, 1995, pp. 293-304.

[25] Soh L. K. \& Tsatsoulis C., Texture Analysis of SAR Sea Ice Imagery using Gray Level Co-occurrence Matrices, IEEE Transactions on Geoscience and Remote Sensing, Vol. 37, No. 2, March, 1999, pp. 780-795.

[26] Gong P. \& Howarth P. J., Frequency-Based Contextual Classification and Gray-Level Vector Reduction for Land-Use Identification, Photogrammetric Engineering and Remote Sensing, Vol. 58, No. 4, April, 1992, pp. 423-437. 